\title{
LETTERS
}

\section{Lulu and Nana open Pandora's box far beyond Louise Brown}

I am responding to the recent CMAJ commentary by Dr. Bartha Maria Knopper and Erika Kleiderman. ${ }^{1}$ The genomics revolution has opened Pandora's box for all life forms and continues to present challenges at every breakthrough. It pushes the boundaries of what is possible and acceptable. For example, today, it is realistic to obtain the complete DNA sequence (genome) of any individual. This linear sequence made up of 4 bases, adenine, thymidine, cytosine and guanine holds the clue to our relationships across the evolutionary tree, including interrelatedness among individuals. It also provides the clues to our differences, including the risks of developing diseases.

The consensus among researchers, clinicians and other professionals is that the "omics" revolution will transform lifetime health care and lifestyle decisions. A global Personal Genome Project network, which currently comprises 5 active Personal Genome Projects in the United States (Boston, since 2005), Canada (Toronto, since 2012), United Kingdom (London, since 2013), Austria (Vienna, since 2014) and China (Shanghai, since 2017) is already underway and has begun to report on valuable findings. The first report on the Canadian Personal Genome project was published in CMAJ in 2018. ${ }^{2}$

To this end, some argue it is desirable to obtain the complete genome sequence of every newborn and use it in dealing with health concerns throughout life. This appears logical given that such individual specific sequences may become a reality for as little as $\$ 100$ in the near future. Determining the complete genome sequence will save diagnostics costs and contain the most relevant information possible.

However, on reflection, this information is bound to have positive as well as negative connotations that will apply to the life of the individual from birth to death. Furthermore, the implications of this information may challenge the rights of parents to give consent on behalf of their child to generate a child's genomic sequence. After all, the child will have to live with the consequences. The lesson for the future - proceed with care.

The most recent dilemma in the genomic revolution was presented by the announcement (Nov. 25, 2018) that a researcher in China had genetically altered a gene in a human embryo that resulted in 2 babies, Lulu and Nana. ${ }^{3}$ Apparently, the twins carry the disabled CCR5 gene that may provide them with protection against HIV infection. They are now test subjects. Unlike the use of gene-editing protocols as therapies to correct genetic alterations in somatic cells, the gene-editing protocol used to generate these twins would make a permanent change to the germ line that could be passed on to future generations.

The births of Lulu and Nana have pushed the boundary of genomic revolution to include generation of genetically engineered babies. This act has been widely condemned as premature, dangerous, alarming and unethical. Given this development, we likely will be hearing of an increasing number of reports on genetically engineered babies in the future. Yet, another woman in China is expecting the birth of a child with genetic modifications. This is new territory.

Like it or not, this development forces us to ask, where do we go from here? Although there has been a failure of selfregulation by the scientific community, it should not lead us to stick our heads in the sand and not consider a more responsible path. The outcry after this announcement is natural and expected. It reminds us of the societal reaction in response to the news of the first heart transplant (1969), first recombinant DNA molecule (1972), first test tube baby (Louise Joy Brown, 1978), first genetically modified organism (1980) and first genetically modified organism food (1994) among others. All of these provoked societal uproar at the time, and brought us to new and uncomfortable territory. Today, they are rather routine and mainstream practices. To the best of my understanding, they have contributed societal good and opened new economies. The question remains, are we overreacting with the birth of the first genetically engineered babies? There is a need for informed reflection.

First, the genetic technology for the creation of genetically engineered babies is freely available, and being used routinely in plants and animals. Furthermore, the technology has been used to treat various genetic disorders affecting different tissue types, including bone marrow, muscle and skin, without affecting reproductive systems. Such practices are now commonplace. The technology to undertake these procedures is relatively easy and does not require expensive laboratories. In fact, it is becoming routine in many research laboratories dealing with genetic methods and almost any laboratory can do it. The births of Lulu and Nana are not a scientific breakthrough. Still, it represents a novelty and a line that has not been crossed before for many reasons.

Second, manipulation of embryos is relatively common in laboratories around the world that deal with reproductive issues. Embryos have been subject to extensive genetic manipulation in many animal species and modified versions of each animal in question have been generated successfully. In addition, human embryos are routinely handled in programs of in vitro fertilization that operate in several countries. Genetically manipulated human embryos have been generated, although to the best of my understanding, modified human embryos have not been implanted for further development and instead are terminated within 14 days. Progression of such embryos beyond this point is banned by all research ethics committees and funding agencies in almost all countries. I do not know of any exception where such a practice is viewed as appropriate.

Third, since its inception, the clustered regularly interspaced short palindromic repeats (CRISPR)/CRISPR associated protein 9 (Cas-9) system (the method used to generate Lulu and Nana) has been recognized as having enormous potential for gene editing in most life forms. The technology however, is still in its infancy and will require substantial research and trials before it could be practised without any 
risk. In addition, its application in altering the genome of a human embryo that results in newborn baby has remained heated in theoretical debates, with some comparing it to the experience with eugenics. Many argue that all genetic modification of human embryos toward production of a baby with a desired genetic alteration, although possible, must remain banned and under strict scrutiny. This technology crosses the boundary of what is acceptable today in society, any society. Apparently, the Chinese researcher who produced Lulu and Nana has been fired from his position. There is a need for extensive societal conversations about the ethics and scientific risks of the applications of this technology.

Finally, the recent report from China, if true, will be the first time genetically manipulated human embryos have been implanted and resulted in live births. The genetic modification introduced in this case is expected to protect the children from HIV infection. If no other changes have resulted from the genetic manipulation (still not known), the girls will develop normally and show protection against HIV infection not unlike vaccination against HIV. More important, they will transmit this trait to their children. Is it ethically acceptable to society given that there are other safe and effective ways to protect people from HIV that do not involve editing an embryo's genes?

The potential for gene editing in humans is enormous. However, the poten- tial for abuse is also substantial. The more important immediate questions deal with several areas of concern. First, there are many unknowns with the outcome that deserve reflection. For example, what do we do with off-target effects? They are bound to happen and will generate undesirable effects. We do not know if Lulu or Nana carry any such effects. How do we deal with the mosaics of unknown effect that will emerge during fetal development? How about the effect of such a modification on future generations? How stable will it be? These questions by no means represent a complete list of serious unknowns that must be dealt in developing any guidelines on this technology.

Probably the most problematic concern is the question of which gene (attribute) to alter? The CCR5 gene used in this attempt does not meet necessary requirements for alteration. ${ }^{4}$ As discussed in recent media coverage, ${ }^{5}$ when polled, nearly $70 \%$ of those asked would support gene editing if it allowed infertile couples to have children or if it allowed a couple to alter a serious disease-causing mutation in an embryo. Respondents were opposed to using it to enhance intelligence quotient or athletic ability, or to change skin colour. Some of these attributes are complex in their determinants. They depend on genes as well as environment. It is easier to enhance them by appropriate environment, nutrition and education. The mechanisms of action of such gene-environment interactions are not understood at present. Such traits should always remain out of bounds of any attempt on genetic engineering of the human germ line.

There is a need for societal discussion toward finding a comfortable place to draw a line and develop mechanisms to obey it. The decision should not be left for researchers and scientists at the bench. It has the potential to affect humanity and must find societal contentment. Otherwise we are left with this key question: Should fears of eugenics stifle scientific progress that may include avoidance of serious genetic diseases from the family lineage?

\section{Shiva M. Singh PhD}

Distinguished university professor, Department of Biology, Western University, London, Ont.

Cite as: CMAJ 2019 June 10;191:E642-3. doi: $10.1503 / \mathrm{cmaj} .71979$

\section{References}

1. Knoppers BM, Kleiderman E. "CRISPR babies": What does this mean for science and Canada? CMAJ 2019;191:E91-2.

2. Reuter MS, Walker S, Thiruvahindrapuram B, et al. The Personal Genome Project Canada: findings from whole genome sequences of the inaugural 56 participants. CMAJ 2018;190:E126-36.

3. Cyranoski D. First CRISPR babies: six questions that remain [news article]. Nature 2018 Nov. 30. doi: 10.1038/d41586-018-07607-3.

4. Arenzana-Seisdedos F, Parmentier M. Genetics of resistance to HIV infection: role of co-receptors and co-receptor ligands. Semin Immunol 2006; 18:387-403.

5. Cyranoski D, Ledford H. Genome-edited baby claim provokes international outcry. Nature 2018; 563:607-8.

Competing interests: None declared. 